\title{
MAGNETIZED LANGMUIR SOLITONS AROUND PULSARS
}

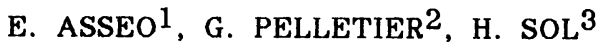 \\ 1 Centre de Physique Théorique, Ecole Polytechnique, \\ 91128 Palaiseau Cedex, France \\ 2 Groupe d'Astrophysique, Observatoire de Grenoble, \\ CERMO BP 68, 38402 Saint Martin D'Heres Cedex, France \\ 3 DARC, Observatoire de Paris, 92195 Meudon Cedex, France
}

\begin{abstract}
Most theoretical works, which aim to interpret the radio radiation observed from pulsars, are based on the idea that coherence of the pulsar emission process could result from the development of plasma instabilities in the magnetosphere of the star. In particular the Ruderman-Sutherland (1975) magnetic pole model reproduces in a satisfying manner several features of the emission. It is based on the development of the two-stream instability. This instability results from the interaction of a beam and a plasma, both in relativistic motion along the open and curved magnetic field lines issued from the magnetic poles of the star. The possibility to obtain radio radiation through this instability process is limited to part of the open cone of magnetic field lines which form an hollow-cone. We describe the linear and nonlinear evolution of the two-stream instability, in the presence of an extremely strong magnetic field. The linear description, including oblique wave propagation (and eventually curvature of the magnetic lines), shows the existence of a natural coupling between the electrostatic and electromagnetic features of the unstable plasma waves which provides the possibility for a direct electromagnetic emission mechanism. The nonlinear evolution of these waves into Langmuir solitons, with also both electrostatic and electromagnetic features, shows the possibility for a new type of radio radiation mechanism, induced by the presence of transverse perturbations of the solitons, which is of interest for the interpretation of pulsar radio observations.
\end{abstract}

\section{Reference}

Ruderman, M.A. and Sutherland, P.G. (1975), Astrophys. J. 196, 51. 